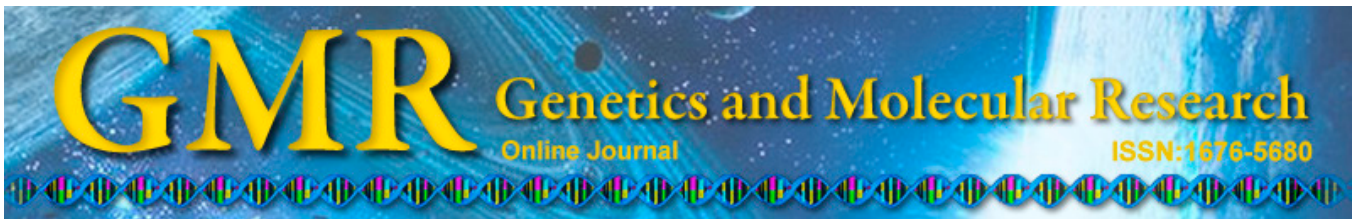

\title{
SAHA attenuates sevoflurane-induced learning and memory impairments in fetal mice
}

\author{
X.F. Lin ${ }^{1}$, Y.Q. Han ${ }^{2}$, H.L. Li ${ }^{3}$, Y.P. Zhao ${ }^{4}$, X.J. Fei ${ }^{5}$ J.X. Sheng ${ }^{4}$, H.H. Lu ${ }^{4}$, \\ S. Liu $^{4}$ and L. Zhang, \\ ${ }^{1}$ Department of Anesthesiology, Huashan Hospital, Fudan University, \\ Shanghai, China \\ ${ }^{2}$ Department of Anesthesiology, Jinan Central Hospital, \\ Shandong University School of Medicine, Jinan, China \\ ${ }^{3}$ Shanghai University of Sport, Shanghai, China \\ ${ }^{4}$ Department of Anesthesiology, East Hospital, \\ Tongji University School of Medicine, Shanghai, China \\ ${ }^{5}$ Department of Hospital Infections, \\ Shanghai Shuguang Hospital Affiliated to Shanghai University of Traditional \\ Chinese Medicine, Shanghai, China \\ ${ }^{6}$ Research Center for Translational Medicine, East Hospital, \\ Tongji University School of Medicine, Shanghai, China \\ Corresponding authors: S. Liu / L. Zhang \\ E-mail: yanglongqiu2@126.com / weiymzhl@126.com
}

Genet. Mol. Res. 13 (4): 10769-10778 (2014)

Received January 22, 2014

Accepted May 8, 2014

Published December 18, 2014

DOI http://dx.doi.org/10.4238/2014.December.18.18

\begin{abstract}
Previous studies have found that children with multiple exposures to anesthesia at an early age are at increased risk of learning and memory impairment. Sevoflurane is the most commonly used inhalational anesthetic for general anesthesia in children. Multiple exposures to sevoflurane have been shown to induce neuroinflammation, inhibit neurogenesis, and cause subsequent learning and memory impairments in fetal mice. Histone-tail acetylation has been implicated in memory formation. In this study, we employed suberanilohydroxamic acid (SAHA), an inhibitor of histone deacetylases, to treat sevoflurane-
\end{abstract}


induced learning and memory impairments. Six-day-old C57BL/6 mice were exposed to sevoflurane for $2 \mathrm{~h}$ daily for 3 days. Morris water maze test performed to evaluate learning and memory impairments and the expression of genes related in to synaptic remodeling/plasticity, or regulated by neuronal activity or the cell cycle were detected by real-time PCR. We found that SAHA attenuated sevoflurane-induced learning and memory impairments in fetal mice. Our findings suggest that SAHA may have potential as a therapeutic agent for preventing or treating the neurotoxicity associated with anesthesia.

Key words: Suberanilohydroxamic acid; Memory impairment; Sevoflurane

\section{INTRODUCTION}

Children with multiple exposures to general anesthesia and surgery at an early age may develop learning disabilities (Wilder et al., 2009; Sun, 2010; Flick et al., 2011). Sevoflurane is the most commonly used inhalational anesthetic for general anesthesia in children. In previous studies, sevoflurane has been shown to inhibit the proliferation of mouse neural progenitor cells, decrease the capacity of neural stem cells for self-renewal, and induce neuroinflammation in microglial cells (Nie et al., 2013; Ye et al., 2013; Zhang et al., 2013a,b). Moreover, in animal studies, multiple exposures to sevoflurane have induced neuroinflammation and inhibited neurogenesis in the brain tissue of 6-day-old fetal mice, and caused subsequent learning and memory impairments 3 weeks later (Lei et al., 2013; Shen et al., 2013). Therefore, the neurotoxicity of sevoflurane is drawing increasing attention in the context of children exposed to inhalational general anesthetics for surgery.

Chromatin modifications, especially histone-tail acetylation, have been implicated in memory formation (Kurdistani and Grunstein, 2003; Goldberg et al., 2007). Acetylation and deacetylation of nucleosomal core histones are important for modulation of chromatin structure and regulation of gene expression (Alarcón et al., 2004). Upregulation of HDAC may result in aberrantly low histone acetylation and abnormal expression of genes critically involved in learning and memory impairments (Korzus et al., 2004). Increased histone-tail acetylation induced by inhibitors of HDAC has been shown to facilitate learning and memory in wild-type mice as well as in mouse models of neurodegeneration (Kumar et al., 2005; Park et al., 2013).

Suberanilohydroxamic acid (SAHA) is a member of a larger class of compounds that inhibit HDAC and is a clinically approved agent. It has been used to treat Sézary syndrome, a type of lymphoma closely related to cutaneous T cell lymphoma (Hockly et al., 2003). A recent study suggesting that SAHA also exerts some activity against recurrent glioblastoma multiforme reported a median overall survival of 5.7 months, compared to the values 4-4.4 months reported in earlier studies (Iwamoto et al., 2013). SAHA can cross the blood-brain barrier, as shown indirectly by measuring changes in histone acetylation in the brain and directly by measuring the amount of SAHA in the brain (Tsankova et al., 2006). We, therefore, tested whether SAHA could ameliorate sevoflurane-induced learning and memory impairments in fetal mice.

In this study, we used 6-day-old mice as a model for investigating the effects of SAHA on the learning and memory impairments induced by sevoflurane. We found that SAHA attenuated sevoflurane-induced learning and memory impairments in these mice. 


\title{
METHODS
}

\section{Animals}

Six-day-old mice were obtained from the specific pathogen free (SPF) animal center at the Shanghai East Hospital affiliated with Tongji University. All animal experiments were approved by the Animal Care Committee of the East Hospital affiliated with Tongji University.

The mice were administered sevoflurane at postnatal day 6 (P6) or from P6 to P8, and then behavioral testing was performed from P31 to P35 or P37. The mice were administered anesthetic (sevoflurane) plus $60 \%$ oxygen (balanced with nitrogen) as performed in previous studies. The $60 \%$ oxygen maintains sufficient partial pressure of oxygen in the mice during anesthesia. Control groups received $60 \%$ oxygen at an identical flow rate in similar chambers. No significant difference was observed in learning and memory functions between mice that received $60 \%$ oxygen and those that received $21 \%$ oxygen (data not shown). The concentrations of the anesthetic and oxygen were measured continuously (Ohmeda, GE Healthcare, Tewksbury, MA, USA). The temperature of the anesthetizing chamber was controlled to maintain a rectal temperature of $37^{\circ} \pm 0.5^{\circ} \mathrm{C}$ in the mice. Previous studies (Shen et al., 2013; Zhang et al., 2013a,b) have shown that anesthesia with 3\% sevoflurane for two hours did not significantly change the values of $\mathrm{pH}$, partial pressure of oxygen, or partial pressure of carbon dioxide in anesthetized mice compared with the control group. Furthermore, compared with control mice, the anesthetized mice did not show significant changes in behavior after anesthesia (e.g., eating, drinking, general activity, and body weight). Mortality rate for mice in these studies was less than $1 \%$. For the intervention studies, SAHA (SML0061; Sigma-Aldrich Shanghai Trading Co. Ltd., Shanghai, China) was dissolved in DMSO as a stock solution and diluted in saline just before injection. SAHA $(25 \mathrm{mg} / \mathrm{kg})$ was administered to mice via intraperitoneal injection $1 \mathrm{~h}$ before each dose of sevoflurane anesthesia for three days.

\section{Morris water maze test (MWM)}

The MWM was conducted in a circular tank (diameter $1.8 \mathrm{~m}$ ) filled with opaque water. A platform $(11 \times 11 \mathrm{~cm})$ was submerged below the water surface in the center of the target quadrant. The swimming path of the mice was recorded using a video camera, and analyzed using the Videomot 2 software (TSE). For each training session, the mice were placed into the maze consecutively from four random points in the tank. Mice were allowed to search for the platform for $60 \mathrm{~s}$. If the mice did not find the platform within $60 \mathrm{~s}$, they were gently guided towards it. Mice were allowed to remain on the platform for $15 \mathrm{~s}$. Two training trials were given every day; the latency for each trial was recorded for analysis. During the memory test (probe test), the platform was removed from the tank, and the mice were allowed to swim in the maze for $60 \mathrm{~s}$.

\section{Real-time polymerase chain reaction (PCR)}

\author{
Real-time PCR was carried out using SYBR-Green-based reagents (EXPRESS
}


SYBR $^{\circledR}$ GreenER ${ }^{\mathrm{TM}}$, Invitrogen) using a CFX96 ${ }^{\mathrm{TM}}$ Real-Time PCR Detection system (Bio$\mathrm{Rad})$. The relative quantities of amplified product were calculated using the comparative Cycle threshold $(\mathrm{Ct})$ method. The results obtained were compared to standard curves generated using serial dilutions of input DNA. Data were derived from three independent amplifications. Error bars represent standard deviations. The primer sequences used for real-time PCR are listed in Table 1. Beta-actin was used as a reference gene (Table 1).

Table 1. Primer sequences used for real-time PCR.

\begin{tabular}{|c|c|}
\hline Primer & Sequence $\left(5^{\prime}-3^{\prime}\right)$ \\
\hline \multirow[t]{2}{*}{ Creb } & 5'-CTACACCAGCTTCCCCGGT-3' \\
\hline & 5'-ACGGAAACAGCCGAGCTC-3' \\
\hline \multirow[t]{2}{*}{$\mathrm{Cbp}$} & 5'-CGGGCAGGGGATGAG-3' \\
\hline & 5'-GCGAGCCAGCGAGGA-3' \\
\hline \multirow[t]{2}{*}{ Nrxn 1} & 5'-CAGGGCCTTTGTCCTGAATA-3' \\
\hline & 5'-GCTTTGAATGGGGTTTTGAG-3' \\
\hline \multirow[t]{2}{*}{ Nrxn 3} & 5'-ACTGAGAGCTAGCCACCCAGAC-3' \\
\hline & 5'-TTGCCCATTTGTGAATTTGA-3' \\
\hline \multirow[t]{2}{*}{$P g k 1$} & 5'-ACATTTTGGCAACACCGRGAG-3' \\
\hline & 5'-GAAGTAGCACGTCTCACTAGTCTCGTG-3' \\
\hline \multirow[t]{2}{*}{ Atf4 } & 5'-GTGATAACCTGGCAGCTTCG-3' \\
\hline & 5'-GGGGTAACTGTGGCGTTAGA-3' \\
\hline \multirow[t]{2}{*}{$p 21$} & 5'-CCACAGTTGGTCAGGGACAG-3' \\
\hline & 5'-СССТCCСCTCTGGGAATCTA-3' \\
\hline \multirow[t]{2}{*}{ Egrl } & 5'-GTGCCCACCACTCTTGGAT-3' \\
\hline & 5'-CGAATCGGCCTCTATTTCAA-3' \\
\hline \multirow[t]{2}{*}{ Egr2 } & 5'-GGCTGCAAATCGTTCCTG-3' \\
\hline & 5'-TCGGAGTATTTATGGGCAGGT-3' \\
\hline \multirow[t]{2}{*}{$c-F O S$} & 5'-GAAAGCCTGGGGCGTAGAGT-3' \\
\hline & 5'-CCTCAGCTGGCGCCTTTAT-3' \\
\hline \multirow[t]{2}{*}{ CamKII $\alpha$} & 5'-GACCTGGATGCTGACGAAG-3' \\
\hline & 5'-AGGTGATGGTAGCCATCCTG-3' \\
\hline \multirow[t]{2}{*}{ Cpgl5 } & 5'-GCGAGATTTCGTTGAGATCG-3' \\
\hline & 5'-GGGATGACACGGATTGATTTT-3' \\
\hline \multirow[t]{2}{*}{ Agrin } & 5'-TTGTAACCAACAGGGGTTGC-3' \\
\hline & 5'-AGTTGTGGCTAGGGGAGCAC-3' \\
\hline \multirow[t]{2}{*}{ Psd95 } & 5'-CCСCTACCCCTCCTGAGAAT-3' \\
\hline & 5'-GAGGGGAAGGAGAAGGTTGG-3' \\
\hline \multirow[t]{2}{*}{ Homerl } & 5'-CTGCCTGAGTGTCGTGGAAG-3' \\
\hline & 3'-ATGATTTCACTCGCGCTGAC-3' \\
\hline \multirow[t]{2}{*}{$C d k 5$} & 5'-CGCAGCCTGTTGGACTTTGT-3' \\
\hline & 3'-GCGTTGCAGAGGAGGTGGTA-3' \\
\hline \multirow[t]{2}{*}{ Shank3 } & 5'-TTTTCCAGGTCCCAGTGGTG-3' \\
\hline & 5'-CCTGCCCACAGTGTCACTCC-3' \\
\hline \multirow[t]{2}{*}{$S v p$} & 5'-CTAGCCTCCCGAATGGAATG-3' \\
\hline & 5'-CAGCAGCAGCATCAGCAATG-3' \\
\hline \multirow[t]{2}{*}{$A r c$} & 5'-CAGCATAAATAGCCGCTGGT-3' \\
\hline & 5'-GAGTGTGGCAGGCTCGTC-3' \\
\hline \multirow[t]{2}{*}{ Synapsin 2} & 5'-GGCTTTCCTTCCCTCCACAC-3' \\
\hline & 5'-TGTTAGCGAGGGAGCAGTGG-3' \\
\hline \multirow[t]{2}{*}{ SNK } & 5'-TTTCCCACGTCCAAAGTCAG-3' \\
\hline & 5'-GCAGCGAAGCTTTAAATACGC-3' \\
\hline \multirow[t]{2}{*}{$N R 2 A$} & 5'-TCGGCTTGGACTGATACGTG-3' \\
\hline & 5'-AGGATAGACTGCCCCTGCAC-3' \\
\hline$N R 2 B$ & 5'-CCTTAGGAAGGGGACGCTTT-3' \\
\hline & 5'-GGCAATTAAGGGTTGGGTTC-3' \\
\hline GLUR-1/AMPA 1 & 5'-GGAGGAGAGCAGAGGGAGAG-3' \\
\hline & 5'-TTCCTGCAATTCCTTGCTTG-3' \\
\hline GLUR-2 & 5'-GCGGTGCTAAAATCGAATGC-3' \\
\hline & 5'-ACAGAGAGGGGCAGGCAGT-3' \\
\hline$\beta$-actin & 5'-CCCATCGCCAAAACTCTTCA-3' \\
\hline & 5'-GGCCACTCGAGCCATAAAAG-3' \\
\hline
\end{tabular}




\section{Statistical analysis}

Data for biochemical changes are reported as means \pm SD. Data for changes in escape latency are reported as means $\pm \mathrm{SE}$. The data for platform crossing times were not distributed normally, and therefore are expressed as median and interquartile range. Two-way repeated measures ANOVA with interactions of factors time and group was used to analyze the differences in learning curves (based on escape latency) for the MWM. The MannWhitney $U$ test was used to determine the differences in platform crossing times. A Student two-sample $t$-test was used to determine the differences in gene expression from real-time PCR results. Values of $\mathrm{P}<0.05$ were considered statistically significant. The SAS software version 9.2 (SAS Institute Inc., Cary, NC, USA) was used to analyze the data.

\section{RESULTS}

\section{Multiple exposures of sevoflurane in fetal mice induced learning and memory impairments}

Clinical studies have shown that children who undergo three exposures, but not one exposure, to anesthesia are at risk of developing learning and memory impairments. Therefore, we treated six-day-old mice with sevoflurane for $2 \mathrm{~h}$ daily over 1 or 3 days. This animal model conceptually mimics single versus multiple exposures of anesthesia and allows us to study anesthesia-induced learning and memory impairments. The fetal mice were treated with 3\% sevoflurane anesthesia for $2 \mathrm{~h}$ daily for 3 days from P6-P8 and tested in the MWM from P30-P34. A comparison of the time that each mouse took to reach the platform during reference training (escape latency) showed that there was a statistically significant interaction of time and group based on escape latency in the MWM between the control group and the sevoflurane anesthesia group (Figure 1A) $(\mathrm{P}=0.0257$, two-way repeated measures ANOVA). A comparison of the number of times that each mouse crossed the location of the absent platform at the end of reference training (platform crossing times) indicated that the sevoflurane anesthesia group crossed the platform fewer times compared with the control group (Figure 1B). There was no significant difference in mouse swimming speed between the mice in the sevoflurane anesthesia group and the mice in the control group (data not shown). The expression of genes related to synaptic remodeling/plasticity, or regulated by neuronal activity or the cell cycle, was decreased after sevoflurane treatment, including Egrl, c-Fos, Cpg15, CamKIIa, Creb, Cbp, Nrxn3, genes encoding got the NMDA receptor subunits, p21, Atf4, and Pgk1 (Table 2). These data suggest that multiple exposures of sevoflurane in fetal mice may induce learning and memory impairments in the fetal mice.

\section{SAHA attenuated sevoflurane-induced learning and memory impairments in fetal mice}

SAHA is a clinically approved agent that has been shown to improve learning and memory. Therefore, we tested whether SAHA could ameliorate the sevoflurane-induced learning and memory impairments in the fetal mice. SAHA was administered to mice via intraperitoneal injection $1 \mathrm{~h}$ before each of the three doses of sevoflurane anesthesia. The mice were 
A

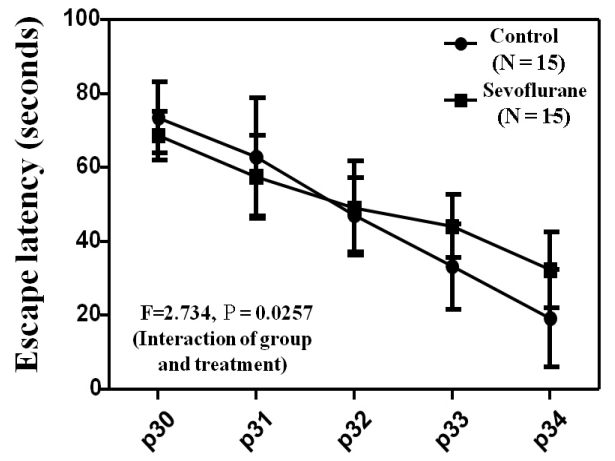

B

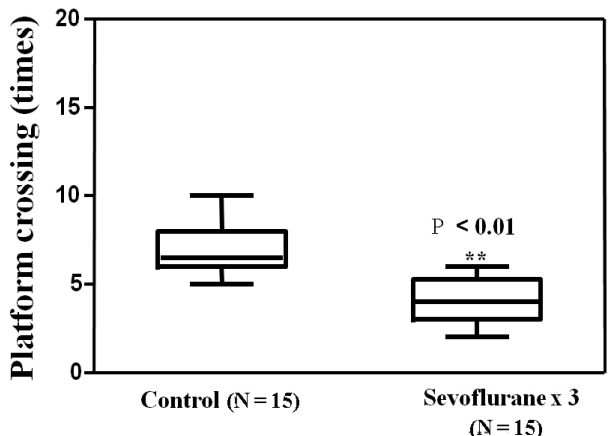

Figure 1. Multiple exposures to sevoflurane in fetal mice induced learning and memory impairments. A. Anesthesia with 3\% sevoflurane for $2 \mathrm{~h}$ daily for 3 days in P6 mice increases the escape latency of mice swimming in the MWM as compared with the control condition (tested from P30-P34) (control, $\mathrm{N}=15$; sevoflurane, $\mathrm{N}=15$ ). Twoway repeated measure ANOVA shows that there is a statistically significant interaction of time and group based on escape latency of MWM between the control group and the sevoflurane anesthesia group. B. Anesthesia with $3 \%$ sevoflurane for $2 \mathrm{~h}$ daily for 3 days in P6 mice decreases the number of platform crossings (platform crossing times) of mice swimming in the MWM compared with the control group tested at P34 (control, $\mathrm{N}=15$; sevoflurane, $\mathrm{N}=$ 15). $* \mathrm{P}<0.05, * * \mathrm{P}<0.01 ; \mathrm{MWM}=$ Morris water maze.

Table 2. Changes in hippocampal gene expression after sevoflurane treatment in mice (levels in the sevoflurane group/levels in the control group).

\begin{tabular}{|c|c|c|c|}
\hline Gene name & Protein name & Fold $\Delta$ & $P$ value \\
\hline CREB & cAMP response element-binding protein & -3.275 & $<0.01$ \\
\hline CBP & CREB-binding protein & -4.57 & $<0.05$ \\
\hline Neurexin I & Neurexin I & -1.374 & $<0.05$ \\
\hline Neurexin III & Neurexin III & -3.752 & $<0.05$ \\
\hline PGK1 & Phosphoglycerate kinase 1 & -6.387 & $<0.01$ \\
\hline ATF4 & Activating transcription factor 4 & -4.274 & $<0.01$ \\
\hline CaMKIIA & Calcium/calmodulin-dependent protein kinase II alpha & -2.484 & $<0.05$ \\
\hline P21 & Cyclin-dependent kinase inhibitor $1 \mathrm{~A}$ & -2.375 & $<0.05$ \\
\hline EGR1 & Early growth response 1 & -3.573 & $<0.05$ \\
\hline EGR-2 & Early growth response 2 & -4.754 & $<0.01$ \\
\hline Agrin & Agrin & -5.356 & $<0.01$ \\
\hline GluR-1 & Glutamate receptor 1 & -2.375 & $<0.05$ \\
\hline GluR-2 & Glutamate receptor 2 & -3.756 & $<0.01$ \\
\hline PSD95 & Postsynaptic density protein 95 & -4.586 & $<0.01$ \\
\hline HOMER1 & Homer protein homolog 1 & -3.284 & $<0.05$ \\
\hline CDK5 & Cyclin-dependent kinase 5 & -7.451 & $<0.01$ \\
\hline SHANK3 & SH3 and multiple ankyrin repeat domains 3 & -3.485 & $<0.01$ \\
\hline SVP & Short vegetative phase & -4.385 & $<0.01$ \\
\hline Synapsin II & Synapsin II & -5.346 & $<0.01$ \\
\hline $\mathrm{ARC}$ & Activity-regulated cytoskeleton-associated protein & -4.38 & $<0.01$ \\
\hline FOS & FBJ osteosarcoma oncogene & -3.485 & $<0.01$ \\
\hline CPG15 & Cuticular protein glycine-rich 15 & -2.385 & $<0.05$ \\
\hline SNK & Serum-inducible protein kinase & -4.342 & $<0.01$ \\
\hline NR2A & $\mathrm{N}$-methyl-D-aspartate receptor subunit 2A & -6.483 & $<0.01$ \\
\hline NR2B & $N$-methyl-D-aspartate receptor subunit $2 \mathrm{~B}$ & -5.385 & $<0.01$ \\
\hline
\end{tabular}

Data are reported as means $\pm \mathrm{SD}, \mathrm{N}=3$.

treated with 3\% sevoflurane for $2 \mathrm{~h}$ daily for 3 days (from P6-P8). Finally, the mice were tested in the MWM from P30-P34. There was no significant difference in escape latency and platform crossing times between the SAHA group and the control group (Figure 2A, B). Two-way 
repeated measures ANOVA showed that there was a statistically significant interaction of time and group based on escape latency of MWM between the sevoflurane anesthesia group and the sevoflurane anesthesia plus SAHA group (Figure 2C) $(\mathrm{P}=0.037)$. A comparison of the number of times that each mouse crossed the location of the absent platform at the end of reference training (platform crossing times) indicated that the sevoflurane anesthesia group crossed the platform fewer times compared with the sevoflurane anesthesia plus SAHA group (Figure 2D). The genes related to synaptic remodeling/plasticity, or regulated by neuronal activity or the cell cycle, were increased in the sevoflurane anesthesia plus SAHA group compared with the sevoflurane anesthesia group, including Bdnf promoter I/II, Egrl, c-Fos, Cpg15, CamKIIa, Creb, Cbp, Nrxn3, genes encoding the NMDA receptor subunits, p21, Atf4, and Pgk1 (Table $3)$. These data suggested that SAHA attenuated sevoflurane-induced learning and memory impairments in fetal mice.

A

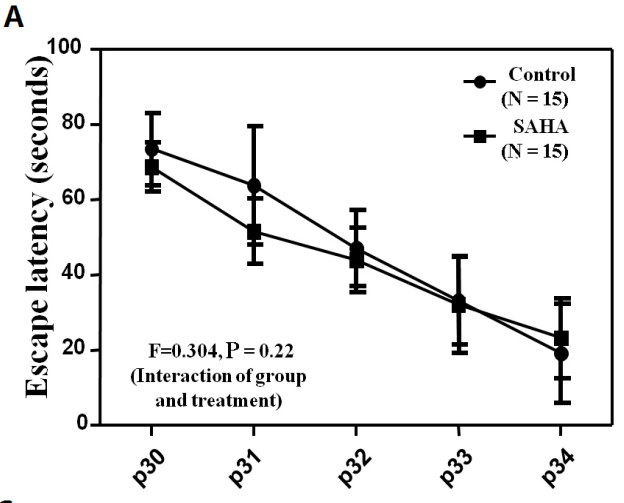

C

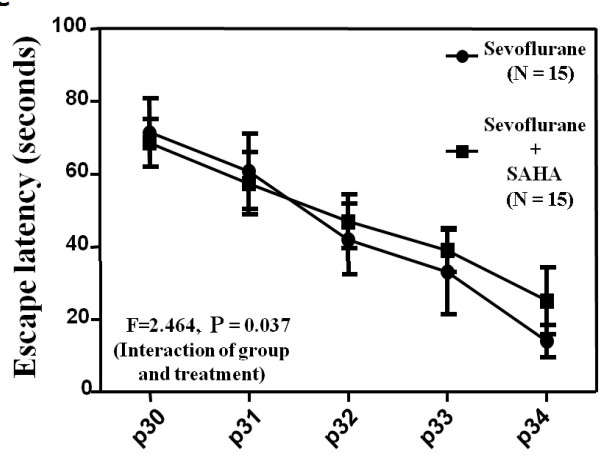

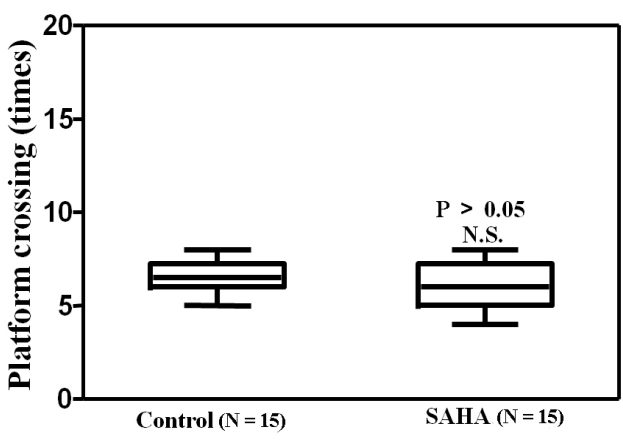

D

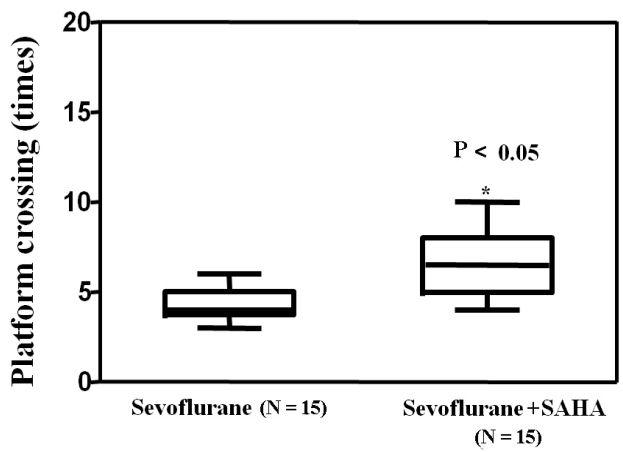

Figure 2. SAHA attenuated sevoflurane-induced learning and memory impairments in fetal mice. A. and B. There was no significant difference in escape latency and platform crossing times between the SAHA group and the control group. C. Two-way repeated measure ANOVA showed that there was a statistically significant interaction of time and group based on escape latency of MWM between the sevoflurane anesthesia group and the sevoflurane anesthesia plus SAHA group $(\mathrm{P}=0.037)$. D. A comparison of the number of times that each mouse crossed the location of the absent platform at the end of reference training (platform crossing times) indicated that the platform crossing times in the sevoflurane anesthesia group were decreased compared with the sevoflurane anesthesia plus SAHA group. ${ }^{*} \mathrm{P}<0.05,{ }^{*} \mathrm{P}<0.01 ; \mathrm{MWM}=$ Morris water maze. 
Table 3. Changes in hippocampal gene expression after sevoflurane and SAHA treatment in mice (levels in the sevoflurane + SAHA group/levels in the sevoflurane group).

\begin{tabular}{|c|c|c|c|}
\hline Gene name & Protein name & Fold $\Delta$ & $P$ value \\
\hline CREB & cAMP response element-binding protein & 1.265 & $<0.05$ \\
\hline CBP & CREB-binding protein & 2.374 & $<0.01$ \\
\hline Neurexin I & Neurexin I & 1.465 & $<0.05$ \\
\hline Neurexin III & Neurexin III & 2.375 & $<0.05$ \\
\hline PGK1 & Phosphoglycerate kinase 1 & 5.375 & $<0.01$ \\
\hline ATF4 & Activating transcription factor 4 & 6.763 & $<0.01$ \\
\hline CaMKIIA & Calcium/calmodulin-dependent protein kinase II alpha & 4.375 & $<0.01$ \\
\hline $\mathrm{P} 21$ & Cyclin-dependent kinase inhibitor $1 \mathrm{~A}$ & 3.576 & $<0.05$ \\
\hline EGR1 & Early growth response 1 & 4.324 & $<0.01$ \\
\hline EGR-2 & Early growth response 2 & 2.485 & $<0.05$ \\
\hline Agrin & Agrin & 4.385 & $<0.01$ \\
\hline GluR-1 & Glutamate receptor 1 & 2.853 & $<0.01$ \\
\hline GluR-2 & Glutamate receptor 2 & 3.475 & $<0.05$ \\
\hline PSD95 & Postsynaptic density protein 95 & 2.475 & $<0.05$ \\
\hline HOMER1 & Homer protein homolog 1 & 3.285 & $<0.01$ \\
\hline CDK5 & Cyclin-dependent kinase 5 & 4.381 & $<0.01$ \\
\hline SHANK3 & SH3 and multiple ankyrin repeat domains 3 & 2.896 & $<0.05$ \\
\hline SVP & Short vegetative phase & 2.143 & $<0.01$ \\
\hline Synapsin II & Synapsin II & 1.472 & $<0.05$ \\
\hline $\mathrm{ARC}$ & Activity-regulated cytoskeleton-associated protein & 7.473 & $<0.01$ \\
\hline FOS & FBJ osteosarcoma oncogene & 3.462 & $<0.01$ \\
\hline CPG15 & Cuticular protein glycine-rich 15 & 1.879 & $<0.05$ \\
\hline SNK & Serum-inducible protein kinase & 4.774 & $<0.01$ \\
\hline NR2A & $N$-methyl-D-aspartate receptor subunit 2A & 5.783 & $<0.01$ \\
\hline NR2B & $\mathrm{N}$-methyl-D-aspartate receptor subunit 2B & 2.785 & $<0.05$ \\
\hline
\end{tabular}

Data are reported as means $\pm \mathrm{SD}, \mathrm{N}=3$.

\section{DISCUSSION}

Previous studies have found that sevoflurane, the most commonly used anesthetic in children, can induce apoptosis, increases in $\beta$-amyloid levels, and neuroinflammation in the brains of fetal mice and caused learning and memory impairment 3 weeks later (Shen et al., 2013; Zhang et al., 2013a,b). Clinical studies have also found that children with multiple exposures to general anesthesia and surgery at an early age may develop learning disabilities (Sun, 2010; Flick et al., 2011). Therefore, the neurotoxicity of sevoflurane is becoming an important issue for parents and anesthetists in the context of children requiring general inhalational anesthesia for surgery.

Learning and memory formation and storage require alterations in gene expression. Chromatin remodeling, especially histone-tail acetylation, alters the compact structure of chromatin and changes the accessibility of DNA to regulatory proteins. Chromatin remodeling is emerging as a fundamental mechanism for regulating gene expression (Vecsey et al., 2007). Histone acetyltransferases add an acetyl group onto the $\varepsilon$-amino group of lysine, thereby reducing the positive charge on histones, which, in turn, decreases their ability to bind to negatively charged DNA; this leads to relaxed chromatin structure and increased accessibility for various transcription factors and other transcription components. Conversely, HDAC remove the acetyl group, thereby restoring the closed chromatin structure (Park et al., 2013). Recently, histone acetylation has been implicated in synaptic plasticity and learning behavior (Vecsey et al., 2007). Moreover, a non-selective HDAC inhibitor was found to reinstate learning ability and promote retrieval of long-term memory in mice, even after massive neuronal loss (Tsankova et al., 2006). Taken together, these observations indicate that HDAC inhibition may provide a potential remedy for memory impairments caused by neurodegenerative and other diseases. 
In this study, we assessed the effects of multiple exposures to $3 \%$ sevoflurane on functional aspects of learning and memory, and brain levels of genes related to memory in mice. We treated 6-day-old mice with 3\% sevoflurane anesthesia for $2 \mathrm{~h}$ daily for 3 days from P6-P8. The mice were tested in the MWM from P30-P34. MWM results showed increased escape latency and reduced platform-crossing times in the sevoflurane-treated group compared with the control group. Moreover, sevoflurane anesthesia downregulated the expression of memory-related genes in the brain tissue. SAHA, one of a larger class of compounds that inhibit HDAC, is used safely in clinical contexts. In our study, we found that SAHA rescued sevoflurane-induced learning and memory impairments and upregulated the expression of memory-related genes in the brain tissues of mice exposed to inhalational anesthesia. These data suggest that SAHA may be a potential therapeutic agent for treating sevoflurane-induced learning and memory impairments.

In conclusion, we found that anesthesia with $3 \%$ sevoflurane for $2 \mathrm{~h}$ daily for 3 days induced impairments in learning and memory in fetal mice. SAHA attenuated sevofluraneinduced learning and memory impairments and may be a potential therapeutic agent for preventing or treating neurotoxicity associated with anesthesia.

\section{REFERENCES}

Alarcón JM, Malleret G, Touzani K, Vronskaya S, et al. (2004). Chromatin acetylation, memory, and LTP are impaired in CBP+/- mice: a model for the cognitive deficit in Rubinstein-Taybi syndrome and its amelioration. Neuron 42: 947-959.

Flick RP, Katusic SK, Colligan RC, Wilder RT, et al. (2011). Cognitive and behavioral outcomes after early exposure to anesthesia and surgery. Pediatrics 128: e1053-1061.

Goldberg AD, Allis CD and Bernstein E (2007). Epigenetics: a landscape takes shape. Cell 128: 635-638.

Hockly E, Richon VM, Woodman B, Smith DL, et al. (2003). Suberoylanilide hydroxamic acid, a histone deacetylase inhibitor, ameliorates motor deficits in a mouse model of Huntington's disease. Proc. Natl. Acad. Sci. U. S. A. 100: 2041-2046.

Iwamoto M, Friedman E, Sandhu P, Agrawal NG, et al. (2013). Clinical pharmacology profile of vorinostat, a histone deacetylase inhibitor. Cancer Chemother. Pharmacol. 72: 493-508.

Korzus E, Rosenfeld MG and Mayford M (2004). CBP histone acetyltransferase activity is a critical component of memory consolidation. Neuron 42: 961-972.

Kumar A, Choi K, Renthal W, Tsankova NM, et al. (2005). Chromatin remodeling is a key mechanism underlying cocaineinduced plasticity in striatum. Neuron 48: 303-314.

Kurdistani SK and Grunstein M (2003). Histone acetylation and deacetylation in yeast. Nat. Rev. Mol. Cell Biol. 4: 276-284.

Lei X, Zhang W, Liu T, Xiao H, et al. (2013). Perinatal supplementation with omega-3 polyunsaturated fatty acids improves sevoflurane-induced neurodegeneration and memory impairment in neonatal rats. PLoS One 8: e70645.

Nie H, Peng Z, Lao N, Dong H et al. (2013). Effects of sevoflurane on self-renewal capacity and differentiation of cultured neural stem cells. Neurochem. Res. 38: 1758-1767.

Park CS, Rehrauer H and Mansuy IM (2013). Genome-wide analysis of H4K5 acetylation associated with fear memory in mice. BMC Genomics 14: 539.

Shen X, Dong Y, Xu Z, Wang H, et al. (2013). Selective anesthesia-induced neuroinflammation in developing mouse brain and cognitive impairment. Anesthesiology 118: 502-515.

Sun L (2010). Early childhood general anaesthesia exposure and neurocognitive development. Br. J. Anaesth. 105: i61-68.

Tsankova NM, Berton O, Renthal W, Kumar A, et al. (2006). Sustained hippocampal chromatin regulation in a mouse model of depression and antidepressant action. Nat. Neurosci. 9: 519-525.

Vecsey CG, Hawk JD, Lattal KM, Stein JM, et al. (2007) Histone deacetylase inhibitors enhance memory and synaptic plasticity via CREB:CBP-dependent transcriptional activation. J. Neurosci. 27: 6128-6140.

Wilder RT, Flick RP, Sprung J, Katusic SK, et al. (2009). Early exposure to anesthesia and learning disabilities in a population-based birth cohort. Anesthesiology 110: 796-804.

Ye X, Lian Q, Eckenhoff MF, Eckenhoff RG, et al. (2013). Differential general anesthetic effects on microglial cytokine expression. PLoS One 8: e52887. 
Zhang L, Zhang J, Yang L, Dong Y, et al. (2013a). Isoflurane and sevoflurane increase interleukin-6 levels through the nuclear factor-kappa B pathway in neuroglioma cells. Br. J. Anaesth. 110: i82-91.

Zhang Y, Dong Y, Zheng H, Shie V, et al. (2013b). Sevoflurane inhibits neurogenesis and the Wnt-catenin signaling pathway in mouse neural progenitor cells. Curr. Mol. Med. 13: 1446-1454. 